\title{
CFD Assessment of Aerodynamic Degradation of a Subsonic Transport Due to Airframe Damage
}

\author{
Neal T. Frink, ${ }^{*}$ Shahyar Z. Pirzadeh,${ }^{\dagger}$ Harold L. Atkins, ${ }^{\ddagger}$ Sally A. Viken, ${ }^{\S}$ Joseph H. Morrison ${ }^{* *}$ \\ NASA Langley Research Center, Hampton, Virginia, 23681
}

\begin{abstract}
A computational study is presented to assess the utility of two NASA unstructured Navier-Stokes flow solvers for capturing the degradation in static stability and aerodynamic performance of a NASA General Transport Model (GTM) due to airframe damage. The approach is to correlate computational results with a substantial subset of experimental data for the GTM undergoing progressive losses to the wing, vertical tail, and horizontal tail components. The ultimate goal is to advance the probability of inserting computational data into the creation of advanced flight simulation models of damaged subsonic aircraft in order to improve pilot training. Results presented in this paper demonstrate good correlations with slope-derived quantities, such as pitch static margin and static directional stability, and incremental rolling moment due to wing damage. This study further demonstrates that highfidelity Navier-Stokes flow solvers could augment flight simulation models with additional aerodynamic data for various airframe damage scenarios.
\end{abstract}

\section{Introduction}

NASA is aggressively engaged in long-term research under the Aviation Safety program to improve the safety of commercial transport aircraft. This research spans a broad scope from integrated systems down through fundamental physics and modeling. Flight safety depends on a complex interaction of integrated systems and is ultimately a matter of maintaining airworthiness and control in the event of onboard system failure, airframe damage, icing, or extreme upset. The Aviation Safety program is structured to address this challenge through four projects: Integrated Vehicle Health Management (IVHM), Integrated Intelligent Flight Deck (IIFD), Integrated Resilient Aircraft Control (IRAC), and Aircraft Aging \& Durability (AAD) ${ }^{1}$.

The present work addresses the resilience of flight control under the Integrated Dynamics and Flight Controls (IDFC) subproject within IRAC. A growing body of wind-tunnel data is available under IRAC/IDFC that measures the static and dynamic aerodynamics and stability \& control (S\&C) on a conventional subsonic transport due to 1) damage to lifting, stability, and control surfaces, 2) icing deformation, and 3) extreme post-stall flight upset conditions. Under IDFC, this dataset is being correlated with computational fluid dynamics (CFD) flow solutions to guide the development of reliable computational tools for predicting and analyzing stability \& control (S\&C) characteristics of aircraft in damaged, icing, and upset flight conditions. The outcome will be calibrated NavierStokes S\&C prediction tools for IDFC flight conditions, along with improved understanding of post-stall aerodynamics of upset aircraft.

In developing "reliable computational tools", it is important to address solution uncertainty, as it will cascade through any subsequent flight simulator model built upon computed aerodynamic data. Solution uncertainty comes from discretization effects from the grids, shortcomings of the turbulence models, and differing methodologies for solving the underlying flow equations. The subject of this paper is a correlation study of two fundamentally different unstructured Navier-Stokes flow solvers, USM3D and FUN3D, with experimental wind-tunnel data [1] for a civilian transport configuration with simulated damage to the wing, vertical tail, and horizontal tail. The objectives are to 1) evaluate the accuracy and utility of the respective numerical tools by correlating with wind-tunnel data for a

\footnotetext{
* Aerospace Engineer, Configuration Aerodynamics Branch, MS 499, Associate Fellow AIAA.

$\dagger$ Aerospace Engineer, Configuration Aerodynamics Branch, MS 499, Senior Member AIAA.

* Aerospace Engineer, Computational AeroSciences Branch, MS 128, Senior Member AIAA.

$\S$ Project Manager/Aerospace Engineer, AvSAFE Integrated Resilient Aircraft Control Project, MS 254, Senior Member AIAA.

** Assistant Head, Computational AeroSciences Branch, MS 128, Senior Member AIAA.

${ }^{1} \mathrm{http}: / /$ www.aeronautics.nasa.gov/programs_avsafe.htm
} 
"damaged" subsonic transport configuration, and then 2) establish application guidelines for simulating aircraft experiencing wing, vertical tail, and horizontal tail damage. The approach is to replicate a substantial subset of the experimentally tested cases from Ref. [1] with progressive losses to the wing, vertical tail, and horizontal tail components. While the results published herein are limited to steady-state solutions, similar work is also underway for dynamic oscillation correlations.

\section{Geometry and Experiment}

The configuration is a representative twin-engine commercial transport configuration called the General Transport Model (GTM) that has received extensive static and dynamic force and moment testing in the NASA Langley 14 by 22 Foot Subsonic Tunnel (14X22). The model span is 82.2 inches, and the mean aerodynamic chord, $m a c$, is 10.98 inches. The tests were conducted at sea level atmospheric conditions with a dynamic pressure of 10 $\mathrm{lb} / \mathrm{ft}^{2}$, corresponding to a Mach number of 0.077 and Reynolds number of $0.54 \times 10^{6}$ based on mean aerodynamic chord. Boundary layer transition strips of width 0.1 inch and number 36 grit were applied at $10 \%$ chord on the upper and lower surfaces of the wing, horizontal and vertical tails. A 0.1 inch wide ring of number 36 grit was applied 1 inch aft of the apex of the nose (measured longitudinally). This gritting pattern was chosen to maintain commonality with previously tested configurations. At the time of this writing it is unclear if this technique was effective in tripping the flow (there was no verification performed, and with the exception of the nose grit, this particular tripping technique differs from well established low-speed practices, e.g. Braslow [2]). However, it was felt that overall trends due to airframe damage, e.g. due to loosing part of the wing, would be worth assessing with fully turbulent CFD even though correlations with absolute levels of the coefficients may not be achieved.

The baseline (undamaged) configuration was tested near the beginning of the NASA Aviation Safety Program to quantify aerodynamic and control characteristics over a broad angle of attack and sideslip range for loss-of-control and flight upset recovery-modeling research. Detailed information on testing conditions, general aerodynamics, and modeling results of the basic configuration are published in Refs. [3-5].

Reference [1] presents the experimental study for the GTM with damage to the left wing, left horizontal tail, and vertical tail. 'Damage' is defined as a partial or total loss of the surface of interest. Types of damage included tip loss (chordwise cuts along the span), trailing-edge surface (flap, elevator, or rudder) loss, and large holes in the surface. The damage configurations studied were tested individually; simultaneous or multiple damage conditions (e.g., a hole combined with tip loss, or combined wing and tail damage) were not evaluated. The model installation with various damaged components is shown in Fig. 1. Additional details of the damage conditions are presented in Fig. 2.

Forced oscillation tests have also been conducted in this facility for the baseline and damaged GTM geometries. The model was oscillated in a sinusoidal fashion at frequencies and amplitudes that correspond to typical full-scale short-period and Dutch-roll motions. Oscillation tests were conducted individually in the pitch and yaw body axes to acquire aerodynamic damping characteristics.

\section{Computational Methods}

Two unstructured Navier-Stokes flow solvers based on fundamentally differing methodologies are exercised in this study. Both codes are being applied to fully tetrahedral unstructured grids. One code (USM3D) solves the flow equations at the cell centers, whereas the other (FUN3D) solves them at the tetrahedral vertices or nodes. The principle issue is that on a given tetrahedral grid, there are between 5 and 6 times more cells than nodes. Hence, the node-centered method will require a finer grid than that needed by a cell-centered code to achieve comparable spatial resolution of the field solution. For this reason, offering an assessment of code-to-code correlations from two fundamentally different methodologies serves to reduce the numerical uncertainty of the results. It does not, however, impact the uncertainty of modeling errors.

\section{A. USM3D ${ }^{2}$}

The cell-centered computations are produced from the USM3D Navier-Stokes flow solver [6] that is part of the NASA Tetrahedral Unstructured Software System (TetrUSS) [7]. USM3D is a parallelized tetrahedral cell-centered, finite volume Navier-Stokes flow solver. The term "cell centered" means that the finite volume flow solution is solved at the centroid of each tetrahedral cell. Inviscid flux quantities are computed across each tetrahedral cell face using various upwind schemes. Spatial discretization is accomplished by a novel reconstruction process, based on an

\footnotetext{
${ }^{2}$ http://tetruss.larc.nasa.gov
} 
analytical formulation for computing solution gradients within tetrahedral cells. The solution is advanced in time by a $2^{\text {nd }}$-order Newton time step scheme, or to a steady-state condition by an implicit backward-Euler scheme. Several turbulence models are available [8]: the Spalart-Allmaras (SA) one-equation model, the two-equation $k$ - $\varepsilon$ turbulence model, the Menter Shear Stress Transport (SST) two-equation model, and the two nonlinear Algebraic Reynolds Stress Models (ARSM) of Girimaji and Shih/Zhu/Lumley. Detached Eddy Simulation (DES) has been implemented in all of the turbulence models. A capability to trip the flow at specified locations on aerodynamic surfaces has been implemented for the $k-\varepsilon$ turbulence model, but fully turbulent flow is assumed for the results to follow. USM3D also has capabilities for dynamic grid motion and overset grids, which are being utilized in dynamic S\&C studies of the GTM to be published in the future.

\section{B. FUN3D ${ }^{3}$}

FUN3D $[9,10]$ is a finite-volume RANS solver based on a node-centered discretization in which the flow variables are stored at the mesh vertices, and control volumes are constructed about each vertex. Inviscid fluxes are approximated by upwind flux-splitting methods using un-weighted least-squares reconstruction techniques. Limiters are applied to the reconstruction for flows with strong shocks. Viscous gradients are evaluated by GreenGauss techniques. The method also supports several turbulence models including Spalart-Allmaras (S-A) oneequation turbulence model [11], and the Mentor SST model [12]. FUN3D supports mixed element grids, including tetrahedra, pyramids, prisms, and hexahedra. The method also supports mesh movement, deformation, overset meshes, and has 6 degrees-of-freedom capability [13,14]. A unique capability of FUN3D is its adjoint capability, which can be used for error estimation, grid adaptation, and design optimization [15]. The current work uses Roe's flux-difference splitting [16] with no limiting applied, and the S-A turbulence model. The flow is assumed to be fully turbulent.

\section{Grid Generation}

The grids have been generated using the unstructured tetrahedral grid generation codes GridTool and VGRID [17], which are part of the NASA TetrUSS system. A developmental version of VGRID [18] was used for the present study.

The primary requirement for comparable grids between a tetrahedral cell-centered (CC) and a node-centered (NC) flow solver are that the numbers and distribution of "cells" for the CC solver be comparable to the numbers and distribution of "nodes" for the NC solver. Such matching between CC and NC grids is difficult to achieve and even more difficult to confirm. For typical tetrahedral grids, there are between 5 and 6 times more cells than nodes, and 2 times more $\mathrm{CC}$ surface triangle faces than $\mathrm{NC}$ surface nodes. Hence, the $\mathrm{NC}$ grid will appear finer than the comparable CC grid. Some commonality is maintained between the USM3D CC and FUN3D NC grids by using the same under lying VGRID input file, but only changing the viscous spacing parameters and a global "inviscid" scaling factor to tailor each grid to the specific flow solver requirements.

\section{A. Grids for USM3D}

Thin-layer tetrahedral grids were generated to meet requirements for cell-centered computations from the USM3D flow solver. The near-wall spacing was prescribed to achieve $y^{+}$of 0.75 in the wall-adjacent tetrahedral cell located near a streamwise distance of $0.5 * \mathrm{mac}$. Since the VGRID Advancing Layers Method [17] marches nodes away from the surface (which are subsequently connected to form cells), a cell-centroid value of $y^{+}=0.75$ is achieved by prescribing an initial "viscous" spacing $\Delta z_{l}$ corresponding to a $y^{+}=3$ of the node. For the GTM at the wind tunnel test conditions, the required initial VGRID spacing is $\Delta z_{l}=0.001239$ inches, and stretching factors of $a=0.15$ and $b=0.02$, where the nodal spacing layers are defined by the Eq. (1). Here " $i$ " is the sequential counter for nodes marching away from the surface.

$$
\Delta z_{i+1}=\Delta z_{1} \cdot\left[1+a \cdot(1+b)^{i}\right]^{i}
$$

This spacing distribution results in approximately 54 tetrahedral cells (18 nodes) across the boundary layer at the mid-chord of the mac. The triangular surface faces were stretched as high as a 10-to-1 ratio along the leading edges of the wing and tails.

\footnotetext{
${ }^{3} \mathrm{http}: / /$ fun3d.larc.nasa.gov
} 


\section{B. Grids for FUN3D}

Grids for FUN3D are generated using the same geometry input and spacing distribution as were used for USM3D grids. Thus the grids are similar in topology. Parametric adjustments are made to VGRID's input in an attempt to account for the differences between cell-centered and node-centered discretizations. The mesh size in inviscid regions is reduced by a factor of $\sim 0.538$ to approximately account for the ratio of nodes to elements. Matching the grids in the viscous layers is more problematic. Adjusting the stretching factors as follows:

$$
\begin{aligned}
& a_{\text {new }}=(1+a)^{(1 / 3)}-1=0.04769 \\
& b_{\text {new }}=(1+b)^{(1 / 3)}-1=0.0066 \\
& \Delta z_{\text {new }, 1}+\Delta z_{\text {new }, 2}+\Delta z_{\text {new }, 3}=\Delta z_{1} \Rightarrow \Delta z_{\text {new }, 1}=0.0003973
\end{aligned}
$$

results in a new grid that has one node for each element of the original grid (or 3 nodes for each "layer" of the original grid.) However, the small value of $b_{\text {new }}$ is problematic for VGRID, and no grids could be generated with this parameter set. By adjusting source strengths in junction regions, it may be possible to generate grids using these parameters; however, this would again alter the overall distribution of nodes and elements, and would defeat the present goal of attempting to match the distribution of the solution variables.

Thus, the approach taken for this study was to hold $b$ fixed, and to reduce $\Delta z_{1}$ and $a$ such that the number of FUN3D solution variables within the boundary layer is close to that of the USM3D on the original grid. In particular for Eq. $1, \Delta z_{1}=0.000109, a=0.10$, and $b=0.02$. It was later determined that, even with this very small initial spacing and reduced stretching, that the mesh size near the edge of the boundary layer at mid-chord is considerable larger than that of the USM3D grid. Ancillary studies, described in the Appendix, indicate that this difference in the wall normal distribution is responsible for differences between USM3D and FUN3D results seen at very high angle of attack.

\section{Assessment of Grid and Turbulence Model Effects}

Prior to this work, an extensive study (yet to be published) was conducted with USM3D to quantify the impact of grid density and turbulence models. Half-span grids were generated on the baseline GTM with 6, 12, 24, 49, and 70 million cells for USM3D. The ranges of turbulence models examined were the SA, SST, k- $\varepsilon$, and Girimaji ARSM. After analysis of the results, the 6 million-cell grid (equivalent to 12 million cells full-span) and the SA turbulence model were selected for the current GTM airframe damage study. Tables 1 and 2 are included below to summarize the effects of grid density and turbulence models on the force and moment coefficients.

Table 1 summarizes the maximum variations of the lift, drag, and pitching moment coefficients $\left(C_{L}, C_{D}\right.$, and $\left.C_{m}\right)$ of the non-selected grids and turbulence models relative to the chosen 6 million-cell (half span) GTM case with the SA turbulence model. The ARSM solutions are not included in the variations due to concerns of poor solution quality. The following relations collect the maximum coefficient variations of all the grids and turbulence models studied relative to the coefficients of the 6 million-cell grid with SA turbulence model.

$$
\begin{aligned}
& \Delta C_{\text {upper }}=\operatorname{MAX}\left\{\operatorname{MAX}\left[\left(C_{12 M}, C_{24 M}, C_{49 M}, C_{70 M}\right)_{S A, S S T, k \varepsilon},\left(C_{6 M}\right)_{S S T, k \varepsilon}\right]-\left(C_{6 M}\right)_{S A}, 0\right\} \\
& \Delta C_{\text {lower }}=\operatorname{MIN}\left\{\operatorname{MIN}\left[\left(C_{12 M}, C_{24 M}, C_{49 M}, C_{70 M}\right)_{S A, S S T, k \varepsilon},\left(C_{6 M}\right)_{S S T, k \varepsilon}\right]-\left(C_{6 M}\right)_{S A}, 0\right\}
\end{aligned}
$$

Note that variations are presented in Table 1 for angles of attack up to 70 degrees, which is beyond the range of 14 degrees addressed in the current study. Large variations are readily observed in Table 1 as angle of attack increases into the stall and post stall regions.

Table 1 - Combined grid and turbulence model effects for USM3D solutions on GTM relative to 6M-cell halfspan (12M cell full-span) grid with SA turbulence model.

\begin{tabular}{|c|c|c|c|c|c|c|}
\cline { 2 - 7 } \multicolumn{1}{c|}{} & Variation from & \multicolumn{5}{c|}{ angle of attack, deg } \\
\cline { 3 - 7 } \multicolumn{1}{c|}{} & 6M, SA & 4 & 10 & 14 & 35 & 70 \\
\hline \multirow{2}{*}{$\Delta C_{L}$} & Upper & 0.0000 & 0.0312 & 0.0052 & 0.0000 & 0.0000 \\
& Lower & -0.0157 & -0.0539 & -0.1666 & -0.1630 & -0.1138 \\
\hline
\end{tabular}

4

American Institute of Aeronautics and Astronautics 


\begin{tabular}{|c|c|c|c|c|c|c|}
\hline \multirow{2}{*}{$\Delta C_{D}$} & Upper & 0.0114 & 0.0126 & 0.0188 & 0.0000 & 0.0000 \\
& Lower & -0.0106 & -0.0165 & -0.0303 & -0.1066 & -0.2950 \\
\hline \multirow{2}{*}{$\Delta C_{m}$} & Upper & 0.0000 & 0.0284 & 0.0088 & 0.2044 & 0.2732 \\
& Lower & -0.0082 & -0.0161 & -0.1824 & 0.0000 & 0.0000 \\
\hline
\end{tabular}

Since Table 1 includes all of the grid and turbulence model effects, Table 2 is provided to isolate the variation due to grid with just the SA turbulence model. The variations are derived from the grid study on the baseline GTM with the SA turbulence model as:

$$
\begin{aligned}
& \Delta C_{\text {upper }}=\operatorname{MAX}\left\{M A X\left[C_{12 M}, C_{24 M}, C_{49 M}, C_{70 M}\right]-C_{6 M}, 0\right\}_{S A} \\
& \Delta C_{\text {lower }}=\operatorname{MIN}\left\{M I N\left[C_{12 M}, C_{24 M}, C_{49 M}, C_{70 M}\right]-C_{6 M}, 0\right\}_{S A}
\end{aligned}
$$

These equations determine the minimum and maximum coefficient variations of the finer grids that were computed with the SA turbulence model from the coefficients of the 6 million-cell grid with SA turbulence model.

Table 2 - Isolated grid effect for USM3D solutions with SA turbulence model on GTM relative to 6M-cell half-span (12M cell full-span) grid.

\begin{tabular}{|c|c|c|c|c|c|c|}
\cline { 2 - 7 } \multicolumn{1}{c|}{} & Variations from & \multicolumn{5}{|c|}{ angle of attack, deg } \\
\cline { 2 - 7 } \multicolumn{1}{c|}{} & 6M, SA & 4 & 10 & 14 & 35 & 70 \\
\hline \multirow{2}{*}{$\Delta C_{L}$} & Upper & 0.0000 & 0.0312 & 0.0000 & 0.0000 & 0.0000 \\
& Lower & -0.0014 & 0.0000 & -0.0236 & -0.0120 & -0.0177 \\
\hline \multirow{2}{*}{$\Delta C_{D}$} & Upper & 0.0000 & 0.0000 & 0.0000 & 0.0000 & 0.0000 \\
& Lower & -0.0106 & -0.0165 & -0.0197 & -0.0105 & -0.0458 \\
\hline \multirow{2}{*}{$\Delta C_{m}$} & Upper & 0.0000 & 0.0000 & 0.0000 & 0.0279 & 0.0319 \\
& Lower & -0.0025 & -0.0153 & -0.0121 & 0.0000 & 0.0000 \\
\hline
\end{tabular}

\section{Results and Discussion}

The effects of airframe damage on the degradation of GTM aerodynamics is addressed in the following subsections. A general discussion on the implications of airframe damage on the aircraft stability and control is presented in Ref. [1]. The focus with the present work is to evaluate the accuracy and utility of the respective numerical tools by correlating with the damaged GTM experimental data from Ref. [1], and establish application guidelines for simulating aircraft experiencing wing, vertical tail, and horizontal tail damage. The following computational results were generated at Mach 0.2 and $\mathrm{Re}_{m a c}=540,000$ with the SA turbulence model assuming fully turbulent flow. Recall that the experimental data employed grit strips for boundary layer tripping, although flow transition has not been verified. The higher Mach number of 0.2 was used in the computations to improve the numerical convergence characteristics of the compressible flow solvers, with the assumption that the fundamental flow physics will not change significantly from Mach 0.077. All computations include the model support sting and were performed in free air, without wind tunnel walls.

\section{A. Horizontal Tail (Stabilizer) Damage}

Tetrahedral grids were constructed on the full span GTM Baseline (No Damage), with progressive horizontal tail (HT) tip losses of $12.5 \%, 25 \%, 50 \%$, "No Left HT", "No HT", and with no elevator control surface on the left HT (see Fig. 2). Figure 3 depicts the surface triangulations for the six USM3D CC grids that range in size from $12,627,932$ cells for the Baseline to 11,025,162 cells for the "No HT" configuration, which are nominally 12 million cells (or 2 million nodes). The grid with no left elevator is not shown in Fig. 3. Similar NC grids were generated for FUN3D on the Baseline and 25\% horizontal tail tip loss cases with nominally 12 million nodes (or 71 million cells). Note that the sting from Fig. 1 is included in the grids.

The impact of horizontal stabilizer damage on longitudinal stability is presented in Figs. 4 and 5. Figure 4 shows a comparison of USM3D (open symbols), and FUN3D (solid symbols) with the 14X22 experimental data (line 
symbols) from Ref. [1]. The CFD correlations with experiment for $C_{m}$ vs. $C_{L}$ appear reasonable, considering the low Mach and Reynolds number of the test data. A good agreement between USM3D and FUN3D results for the Baseline No-Damage and 25-percent tip loss cases brings a level of assurance that the CFD is being solved consistently. The computational results reflect an expected reduction in pitching moment with decreasing horizontal tail area in the low to mid $C_{L}$ range, whereas the experimental data do not for 25- and 50-percent tip losses. Figure 5 reveals a very good correlation of both USM3D and FUN3D with experiment for static margin about the 25-percent $m a c$, derived from the slope of $C_{m}$ vs. $C_{L}$. This is an important result toward meeting the IRAC goal of accurate modeling the $\mathrm{S} \& \mathrm{C}$ characteristics of damaged subsonic transport aircraft.

\section{B. Vertical Tail Damage}

Tetrahedral grids were constructed on the full span GTM Baseline (No Damage), with progressive vertical tail (VT) tip losses of 12.5\%, 25\%, 50\%, and "No VT", and with "rudder off" (see Fig. 2). Figure 6 depicts the surface triangulations for the five USM3D CC grids that range in size from 24,826,975 cells for the Baseline to 23,515,579 cells for the "No VT" configuration, which are nominally 24 million cells (or 4 million nodes). The "rudder off" grid is not shown in Fig. 6, but consists of the full vertical tail minus the rudder. A similar family of five NC grids was generated for FUN3D with nominally 12 million nodes (71 million cell). Time did not permit the regeneration of a series of 12 million-cell CC grids with vertical tail tip loss.

The correlation of USM3D and FUN3D results is presented in Fig. 7 for the effect of vertical tail loss on yawing moment coefficient $\left(C_{n}\right)$ vs. angle of sideslip $(\beta)$ at $\alpha=0$ deg. An excellent correlation with the experimental data is observed for both flow solvers. The effect of vertical tail tip loss is a progressive degradation of directional stability as evidenced by the decrease in positive slope of $C_{n}$ vs. $\beta$ and, as expected, a complete loss of directional stability for the "No VT" case. The important correlation of slope is reflected in the plot of static directional stability $\left(C_{n_{\beta}}\right)$

over the entire angle of attack range in Fig. 8. The experimental $C_{n_{\beta}}$ from Ref. [1] was computed as the linear slope of the yawing moment between sideslip angles of $-4^{\circ}$ and $+4^{\circ}$. Since the numerical results were only computed for negative sideslips and the solutions are strongly symmetric about $\beta=0^{o}$, the $C_{n_{\beta}}$ was computed from the $C_{n}$ at $\beta=-4^{o}$ and an assumed $C_{n}=0$ at $\beta=0^{\circ}$.

\section{Wing Damage}

A set of tetrahedral grids were constructed on the full span GTM Baseline (No Damage), with progressive loss of the left wing tip (WT) by $11 \%, 18 \%, 26 \%, 33 \%$, and $55 \%$ of semispan, and an inboard hole in the left wing panel (see Fig. 2). Figure 9 portrays the surface triangulation for the six USM3D CC grids that range in size from $12,627,932$ cells for the Baseline to $11,055,987$ cells for the $55 \%$ tip loss configuration, which is nominally 12 million cells (or 2 million nodes). A similar family of six NC grids was generated for FUN3D, which had nominally 12 million nodes (or 71 million cells).

The effect of left wing tip loss on the GTM lift coefficient is presented in Fig. 10. The open symbols denote USM3D solutions and solid symbols represent FUN3D results. Both are shown in comparison with 14 X22 wind tunnel data indicated by line symbols. The overall trend of lift loss with increasing wing-tip loss is well captured, but some offset is evident in the absolute levels. A similar comparison is shown in Fig. 11 for rolling moment $\left(C_{\ell}\right)$ vs. angle of sideslip $(\beta)$ at $\alpha=0 \mathrm{deg}$. Again, the trends with increasing wing-tip loss are generally captured, but absolute levels vary slightly.

The resultant roll asymmetry induced by the loss of the left wing tip and an inboard hole is quantified in Fig. 12 for $\beta=0$ deg. Figure 13 shows the total incremental rolling moment $\left(\Delta C_{\ell}\right)$ due to tip loss and $\beta=-4$ deg. (damage wing forward) relative to the undamaged airplane at 0 deg. sideslip. The open symbols denote USM3D solutions and solid symbols represent FUN3D results. As before, the trends in rolling moment increment from left wing damage are well captured, although the computed increments are consistently larger (i.e. more rolling moment) than those measured. Interestingly, the code-to-code agreement between USM3D and FUN3D is very good relative to their absolute levels as compared to experiments, which suggests the numerical results are being computed consistently within the assumptions of a prescribed geometry with fully turbulent flow using the Spalart Allmaras turbulence model. 


\section{Thoughts on CFD Contributions to Flight Simulation Models}

The ultimate goal of this study is to advance the probability of using computational aerodynamic data in the creation of flight simulation models of damaged subsonic transport aircraft. This could require a significant number of numerical solutions of sufficient accuracy

After an extensive assessment of grid and turbulence model effects, full span grids were judged as suitable with 12 million cells for the CC USM3D code, and 12 million nodes for the NC FUN3D code. Also, the well-established SA turbulence model was selected for the computations.

The degree of required accuracy is an ongoing discussion between the CFD and flight simulation communities. From the results presented herein, we have demonstrated that slope-derived quantities, such as pitch static margin and static directional stability $\left(C_{n_{\beta}}\right)$ can be computed with reasonable accuracy, as well as incremental parameters such as rolling moment coefficient $\left(\Delta C_{\ell}\right)$ due to wing damage. The ability to compute absolute levels of coefficients is inconclusive due to the uncertainties associated with comparing to low Mach and Reynolds number wind tunnel data. However, absolute levels of directional stability were well estimated for the baseline GTM with vertical tail losses with a high degree of correlation due to geometric symmetry.

Regarding the ability of CFD to contribute large numbers of solutions, a typical 12 million-cell USM3D solution in this study required $375 \mathrm{CPU}$ hours on an Intel Xeon multiple processor machine. By running each case on 34 processors of a 276-processor cluster dedicated to the IRAC/IDFC project, a typical solution required approximately 11 wall-clock hours to complete. If the entire IRAC/IDFC machine were utilized, then 16 USM3D data points could be generated per day (480 per month). Access to additional computational resources will increase the throughput proportionally. While this is nowhere near the daily productivity of a wind tunnel test, it could be sufficient to augment flight simulation models with additional aerodynamic data for various airframe damage scenarios.

\section{Concluding Remarks}

One of the goals of the IRAC project is to improve aviation safety by providing pilots with more realistic flight simulator training for potential loss-of-control flight events, such as airframe damage, extreme upsets, and icing. In the past, flight simulator models have been built from a composite of experimental data, which is scarce when it comes to airframe damage. One intention of this work is to highlight the potential of using high-fidelity computational tools to generate quality aerodynamic data that may be used to extend flight simulation models of transport aircraft into the realm of airframe damage. The results of this study should serve to advance this dialog with the flight simulation community.

\section{Acknowledgments}

The second author, Dr. Shahyar Z. Pirzadeh passed away unexpectedly on March 18, 2009. As a key member of the IRAC/IDFC CFD team, his contribution in generating the grids was critical to the outcome of this study. Furthermore, Dr. Pirzadeh's many longstanding contributions to the ascension of tetrahedral grid generation methodology as a whole are widely valued throughout the world. We are deeply saddened by this great loss.

\section{References}

1. Shah, Gautam H.: "Aerodynamic Effects and Modeling of Damage to Transport Aircraft", AIAA 2008-6203, August 2008.

2. Braslow, A.L. Hicks, R.M., and Harris, Jr., R.V.: "Use of Grit-Type Boundary Layer-Transition Trips on Wind Tunnel Models", NASA TN D-3579, September 1966.

3. Shah, Gautam H.; Cunningham, Kevin; Foster, John V.; Fremaux, C. Michael; Stewart, Eric C.; Wilborn, James E.; Gato, William; and Pratt, Derek W.: "Wind Tunnel Investigation of Commercial Transport Aircraft Aerodynamics at Extreme Flight Conditions", SAE-2002-01-2912, November 2002.

4. Foster, John V., Cunningham, Kevin, Fremaux, Charles M., Shah, Gautam H., Stewart, Eric C., Rivers, Robert A., Wilborn, James E., and Gato, William: "Dynamics Modeling and Simulation of Large Transport Airplanes in Upset Conditions", AIAA-2005-5933, August 2005.

5. Murch, Austin M. and Foster, John V.: "Recent NASA Research on Aerodynamic Modeling of Post- Stall and Spin Dynamics of Large Transport Airplanes", AIAA-2007-0463, January 2007.

6. Frink, N. T., "Tetrahedral Unstructured Navier-Stokes Method for Turbulent Flows,” AIAA Journal, Vol. 36, No. 11, November 1998, pp. 1975-1982.

7. Frink, N.T., Pirzadeh, S.Z., Parikh, P.C., and Pandya, M.J., "The NASA Tetrahedral Unstructured Software System (TetrUSS)," The Aeronautical Journal, Vol. 104, No. 1040, October 2000, pp. 491-499. 
8. Pandya, M. J., Abdol-Hamid, K. S., and Frink, N. T.: "Enhancement of USM3D Unstructured Flow Solver for HighSpeed High-Temperature Shear Flows", AIAA 2009-1329, January 2009.

9. Anderson, W. K. and Bonhaus, D. L., "An Implicit Upwind Algorithm for Computing Turbulent Flows on Unstructured Grids" Computers and Fluids, Vol. 23, No. 1, 1994, pp. 1-22.

10. Nielsen, E. J., Aerodynamic Design Sensitivities on an Unstructured Mesh Using the Navier-Stokes Equations and a Discrete Adjoint Formulation, Ph.D. thesis, Virginia Polytechnic Institute and State University, Blacksburg, VA, 1998.

11. Spalart, P. R. and Allmaras, S. R., "One-Equation Turbulence Model for Aerodynamic Flows," AIAA Paper 92-0429, 1992.

12. Menter, F. R., "Two-Equation Eddy-Viscosity Turbulence Models for Engineering Applications," AIAA Journal, Vol. 32, No. 8, 1994, pp. 1598-1605.

13. Biedron, R. T., Vatsa, V. N., and Atkins, H. L., "Simulations of Unsteady Flows Using an Unstructured Navier-Stokes Solver on Moving and Stationary Grids," AIAA Paper 2005-5093, June 2005.

14. Biedron, R. T. and Thomas, J. T., "Recent Enhancements to the FUN3D Flow Solver for Moving Mesh Applications," AIAA Paper 2009-1360, Jan. 2009.

15. Nielsen, E. J., Lu, J., Park, M. A., and Darmofal, D. L., "An Implicit, Exact Dual Adjoint Solution Method Implicit, Exact Dual Adjoint Solution Method for Turbulent Flows on Unstructured Grids," Computers and Fluids, Vol. 33, No. 9, 2004, pp. 1131-1155, See also AIAA Paper 2003-0272.

16. Roe, P. L., "Approximate Riemann Solvers, Parameter Vectors, and Difference Schemes," Journal of Computational Physics, Vol. 43, 1981, pp. 357-372.

17. Pirzadeh, S. Z., "Unstructured Viscous Grid Generation by the Advancing-Layers Method," AIAA Journal, Vol. 32, No. 8, pp. 1735-1737, 1994.

18. Pirzadeh, S. Z., “Advanced Unstructured Grid Generation for Complex Aerodynamic Applications,”, AIAA 2008-7178, August 2008.

\section{Appendix}

In most cases of this study, USM3D and FUN3D results were in close agreement; however, at high angle of attack (post stall) some differences were noted. Upon closer examination, it was observed that the FUN3D grid is considerable coarser than the USM3D grid in the region near the edge of the boundary layer $(z=\sim 0.1-0.2$ at the wing mid-chord.) Figure A1 shows the mesh distributions for the "standard" USM3D and FUN3D grids used for this study. Also shown are the "ideal" FUN3D mesh distribution described in the Grid Generation section that closely matches the distribution of the USM3D grid, and an "improved" mesh distribution described below. The abscissa, $d z$, denotes a generalized measure of height per degree of freedom, as opposed to height between nodes, elements, layers, or some other topology dependent description.

The "improved" mesh distribution corresponds to the stretching parameters: $\Delta z_{1}=0.00025, a=0.04769$, and $b=0.015$ ( $a$ matches that of the "ideal" mesh.) Grids were obtained for the baseline (No Damage) geometry after making some minor adjustments to some of the VGRID source strengths, and by making modifications to the VGRID code that alters how the generation of the viscous layers is terminated. In addition, the VGRID inviscid scaling factor, "ifact", was changed after generation of the surface grid in order to better match the solution degrees of freedom on the surface and in the volume (note: this is a highly unusual procedure that works in conjunction with the code modification, and is not generally recommended.) The "improved" mesh better matches the USM3D mesh near the edge of the boundary layer, and is only slightly finer at the surface. However, because $b$ is still larger than the "ideal" value, the wall normal height of the "improved" mesh continues to grow outside of the boundary layer such that the total number of nodes in the mesh is just over 10 million (less than the targeted 12 million cells of the USM3D grid, or the 12 million nodes of the "standard" FUN3D grid.)

Figure A2 shows a plot of $C_{L}$ vs. angle of attack for several solutions of the baseline geometry. In addition to the "standard" grids, FUN3D was run using the "improved" grid in two different modes: all tetrahedron and mixed element. In the latter mode the grid nodes are reconnected to form prismatic layers in the viscous region. This in effect improves the orthogonality of the underlying node-centered stencil without changing the underlying grid. All solutions are in close agreement for an angle of attack $<10$ degrees. At an angle of attack of 14 degrees, the FUN3D solutions on the "improved" mixed-element grid show better agreement with the USM3D solutions. USM3D solution on the baseline geometry are not available for angle of attack of 10 or 12 degrees; however, data from the horizontal tail damage case (not shown) suggest that none of the solutions are in close agreement with one another at an angle of attack of 12 degrees. Thus the agreement at an angle of attack of 14 degrees may be fortuitous. Based on these results, the solutions obtained using the "standard" grids are believed to be mesh independent for angles of attack $<=10$ degrees. 


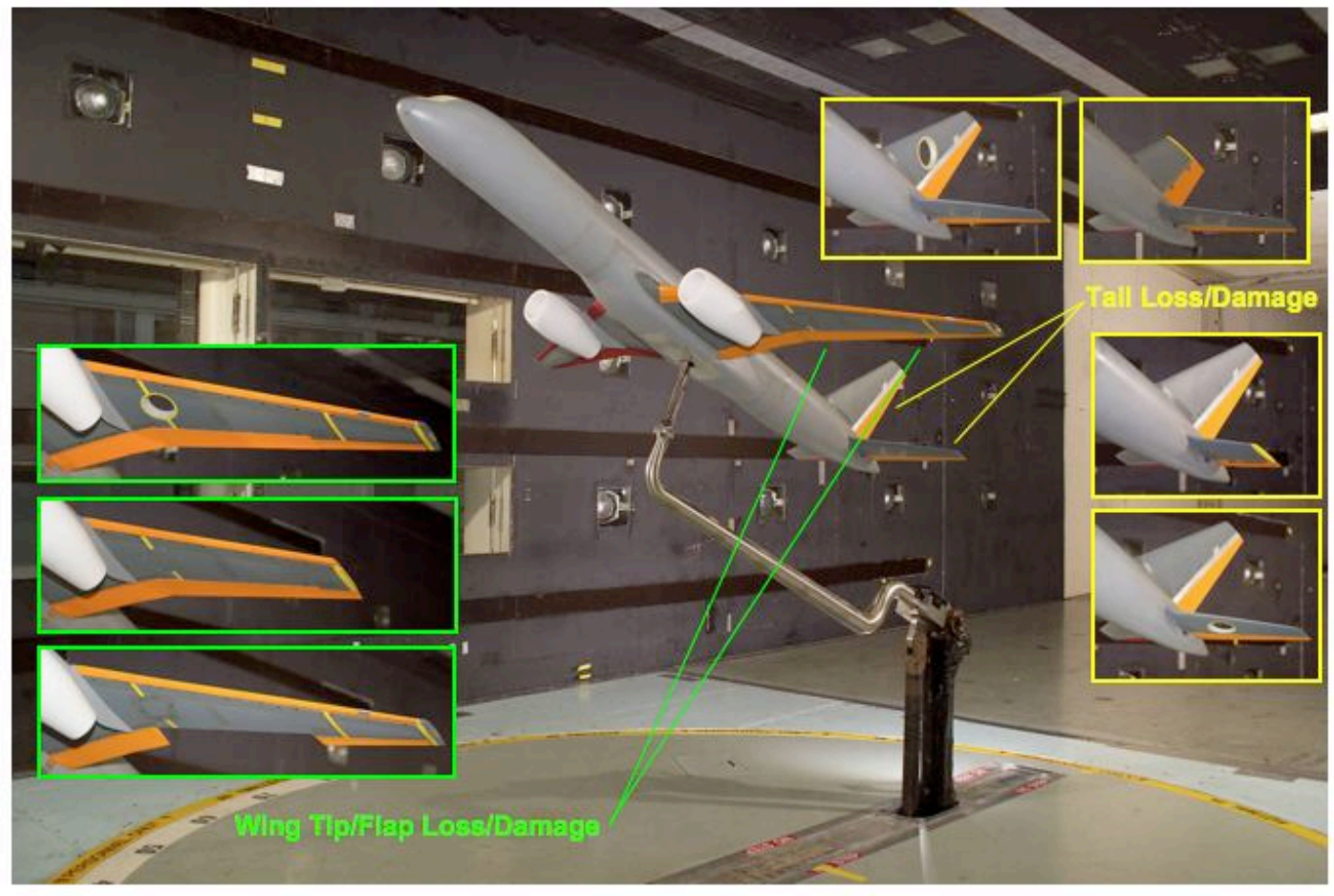

Figure 1. - General Transport Model (GTM) as tested in NASA Langley 14 by 22 Foot Subsonic Tunnel with representative airframe damage.

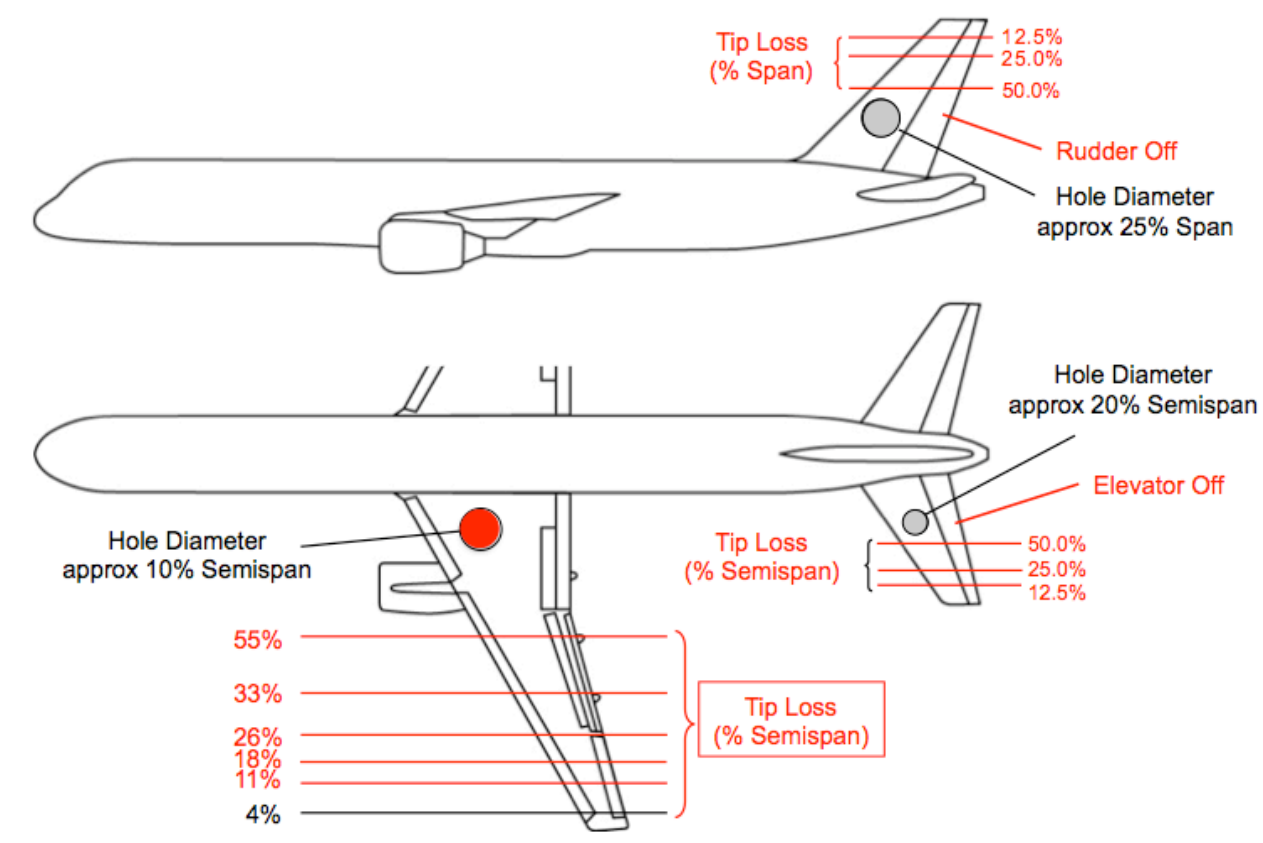

Figure 2. - Sketch of available airframe damage on GTM wind-tunnel configuration. Computed configurations are shown in red. 

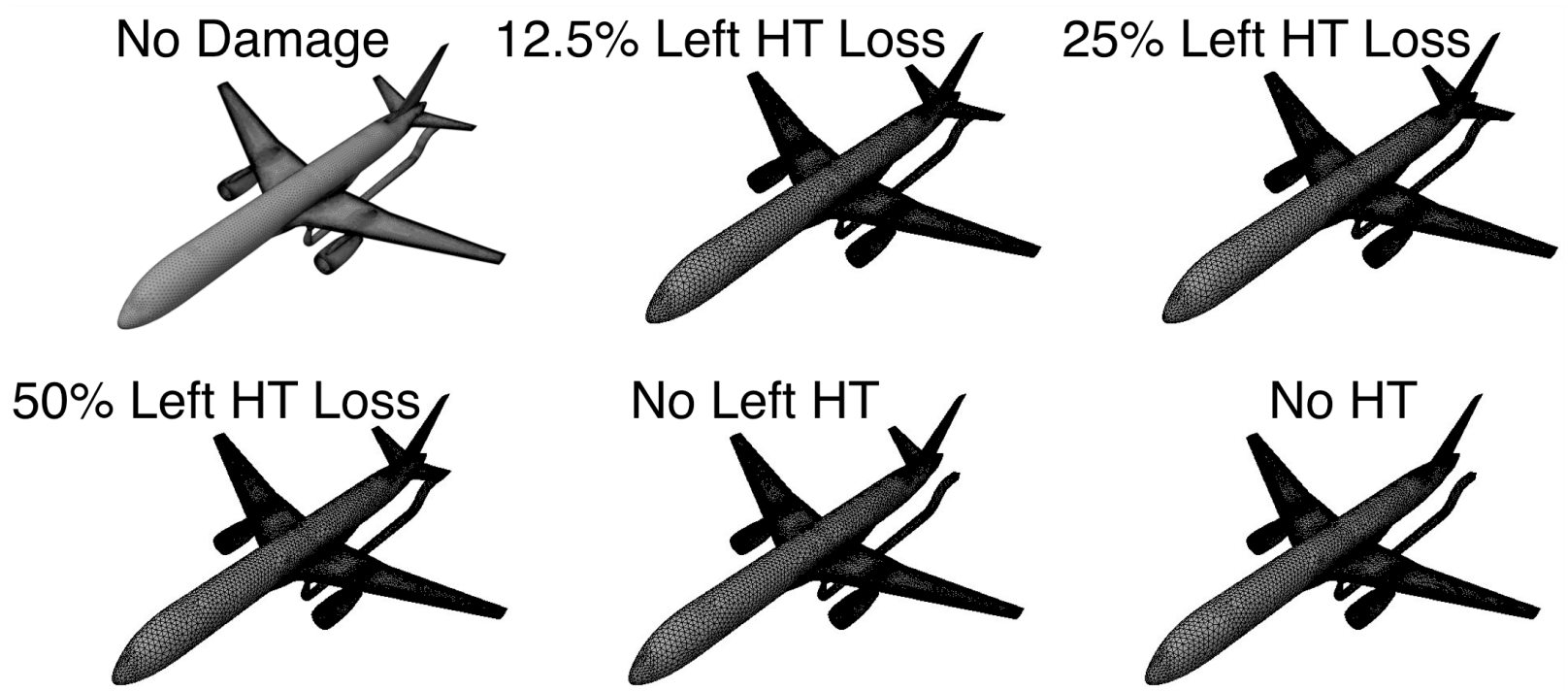

Figure 3. - Surface triangulation of USM3D grids for GTM left horizontal tail (HT) damage configurations. (approx. 12M cells). Note that the model support sting is included in the grids.

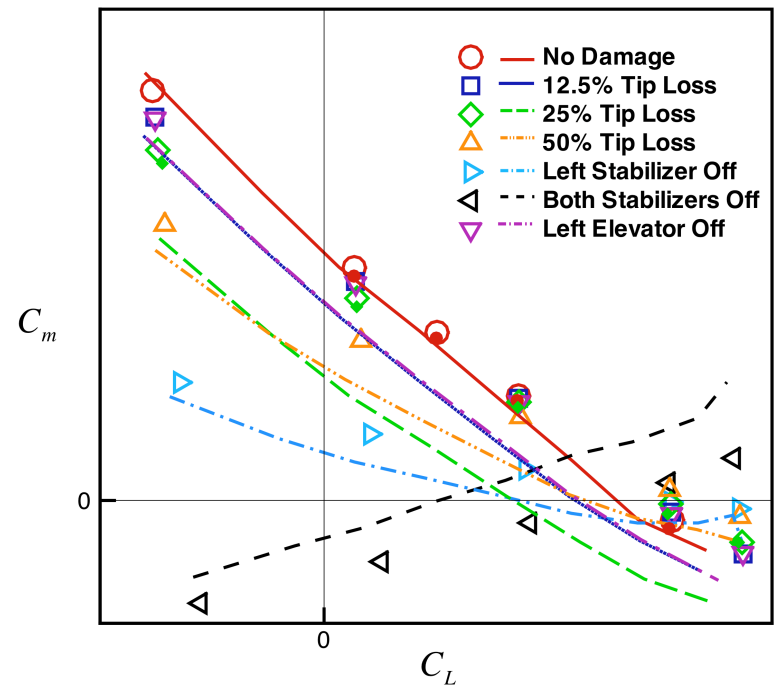

Figure 4. Effect of left horizontal tail damage on pitching moment for GTM. M=0.2, $\beta=0$ deg. USM3D (open symbols), FUN3D (solid symbols), 14X22 WT experiment (line symbols).

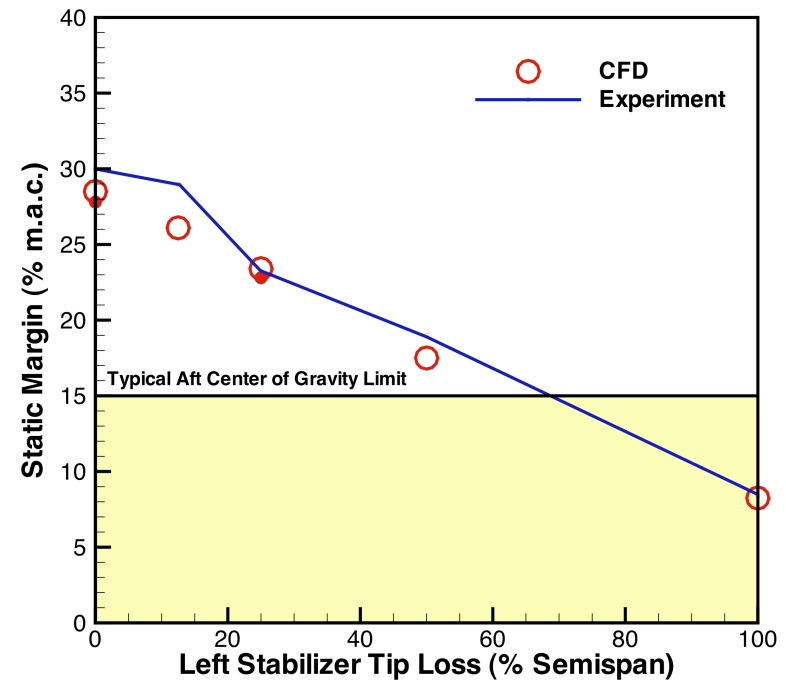

Figure 5. Impact of left horizontal tail damage on static margin of GTM. $M=0.2, \beta=0$ deg. USM3D (open symbols), FUN3D (solid symbols), 14X22 WT experiment (line symbols). 

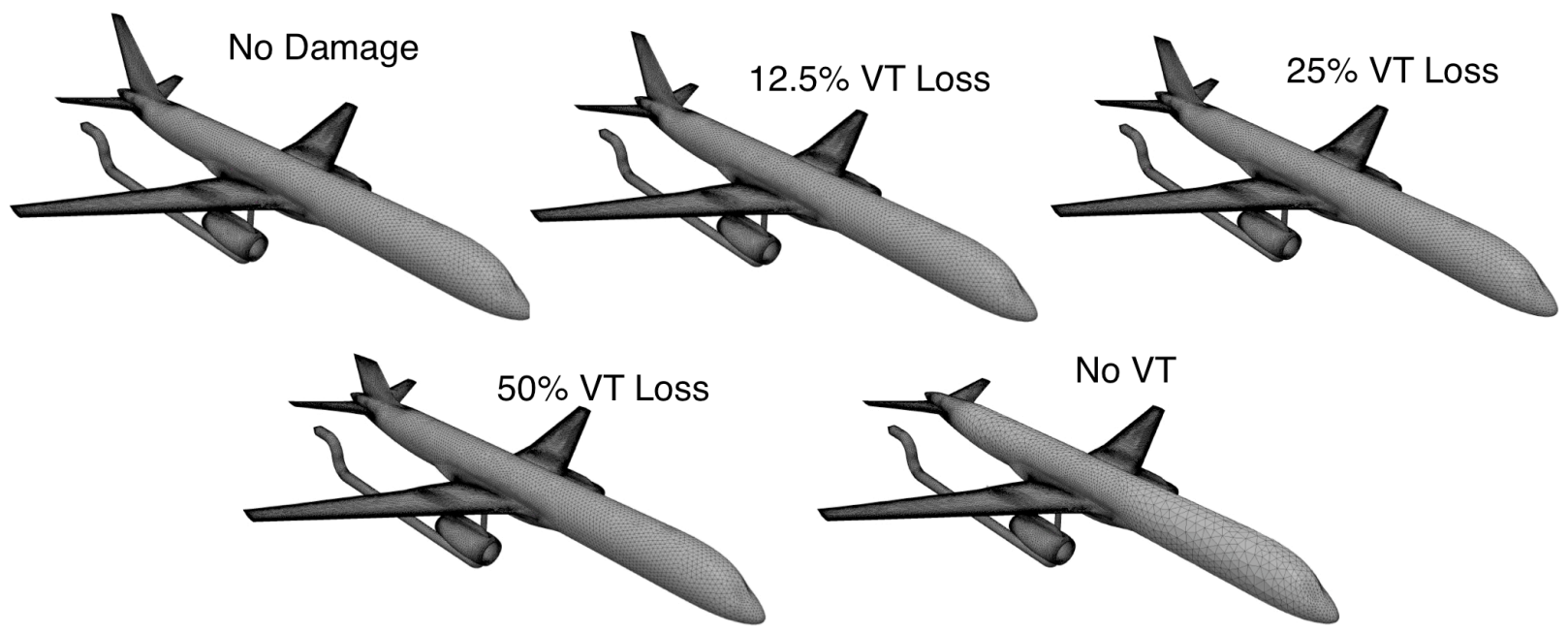

Figure 6. - Surface triangulation of USM3D grids for vertical tail (VT) damage configurations. (approx. 24 million cells)

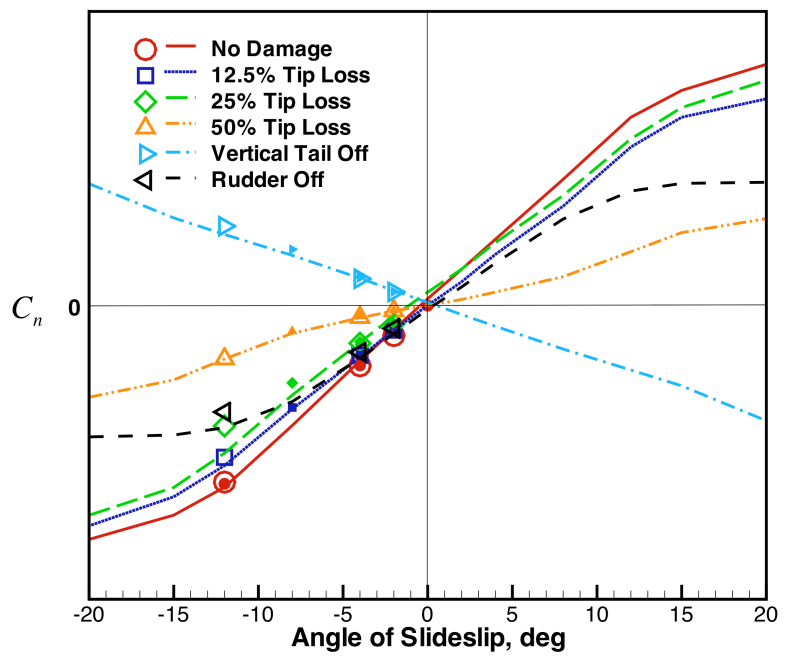

Figure 7. Effect of vertical tail damage on yawing moment for GTM. M=0.2, $\alpha=0$ deg. USM3D (open symbols), FUN3D (solid symbols), 14X22 WT experiment (line symbols).

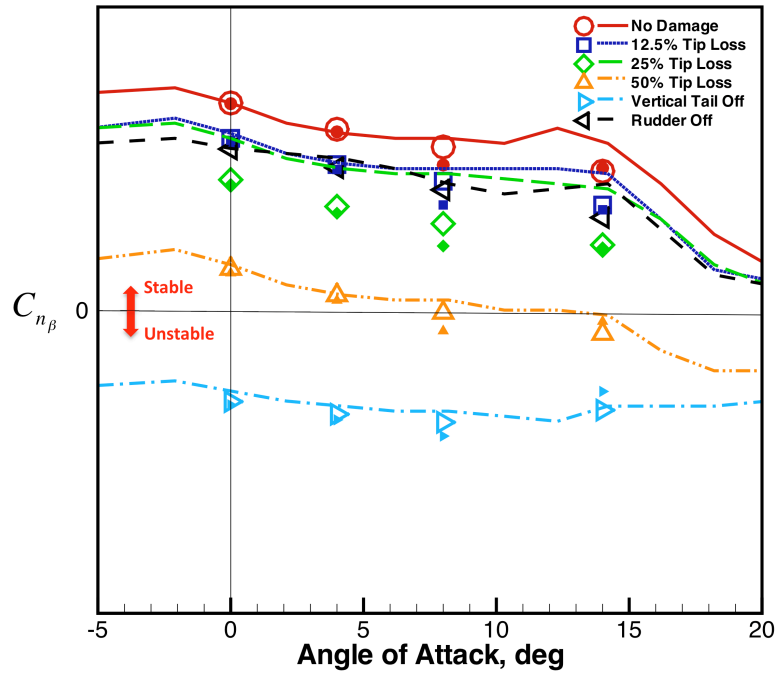

Figure 8. Effect of vertical tail damage on static directional stability for GTM. $M=0.2$, USM3D (open symbols), FUN3D (solid symbols), 14X22 WT experiment (line symbols). 

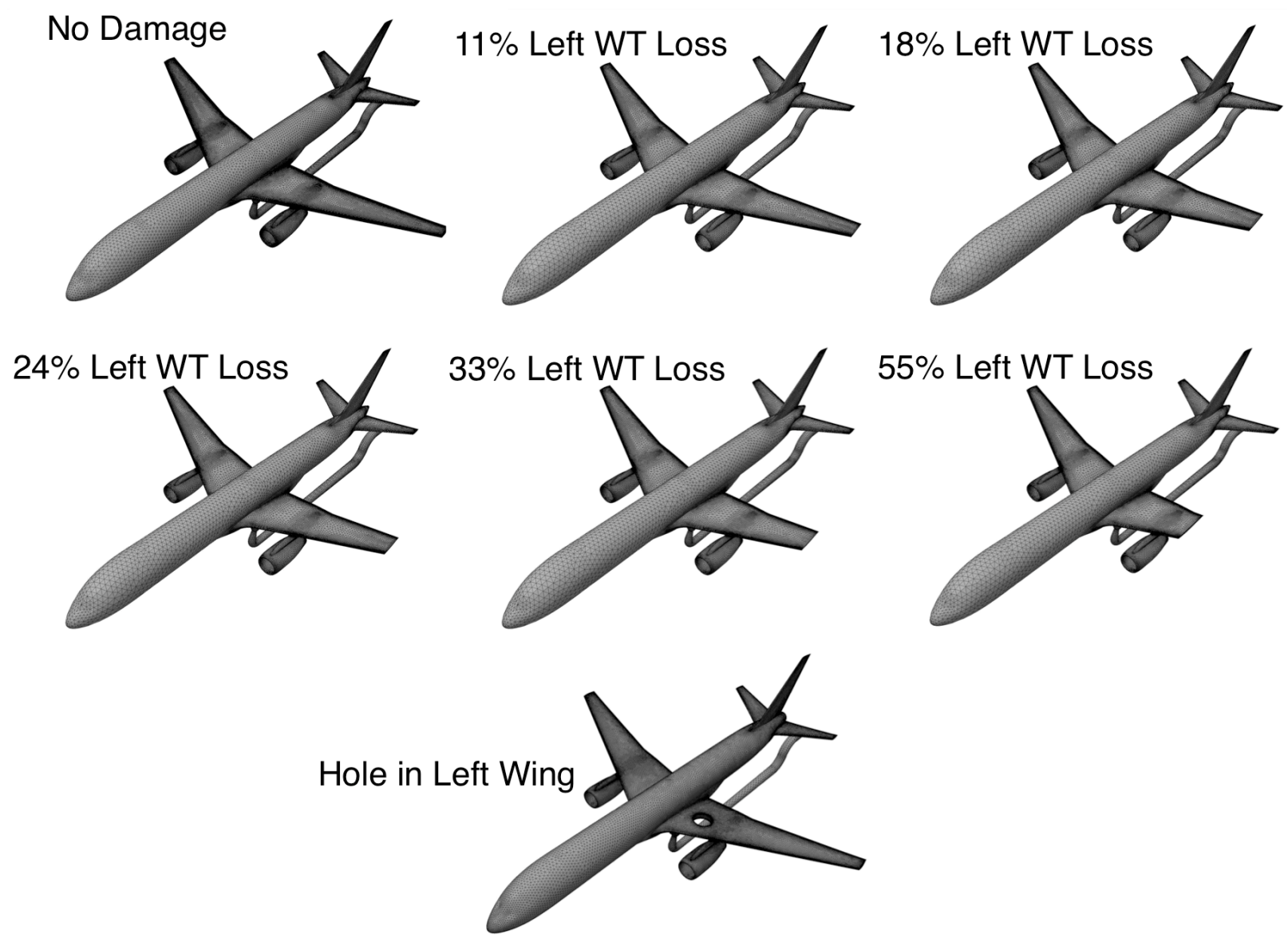

Figure 9. - Surface triangulation of USM3D grids for wing tip (WT) damage configurations. (approx. 12 million cells)

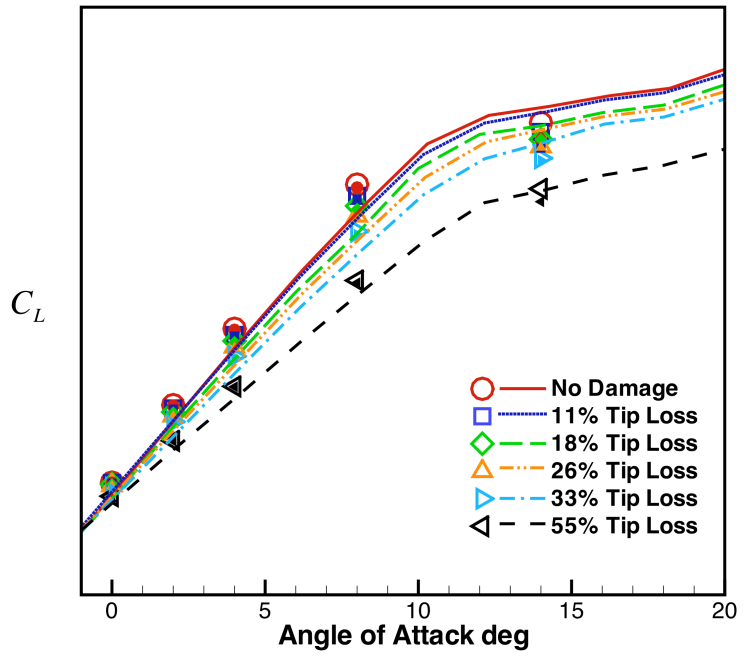

Figure 10. Effect of GTM left wing damage on lift coefficient. $M=0.2, \beta=0$ deg. USM3D (open symbols), FUN3D (solid symbols), 14X22 WT experiment (line symbols).

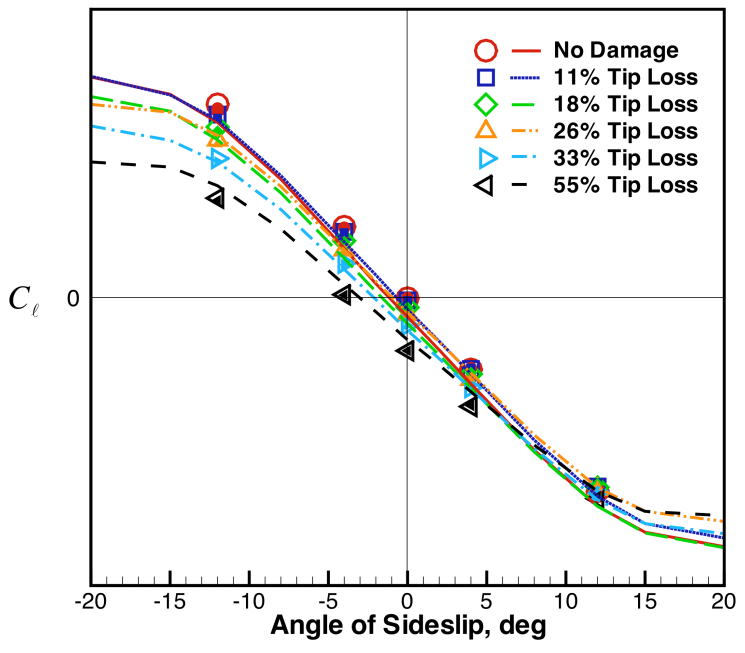

Figure 11. Effect of GTM left wing damage on rolling moment coefficient vs. sideslip. $M=0.2$, $\alpha=0$ deg. USM3D (open symbols), FUN3D (solid symbols), 14X22 WT experiment (line symbols). 


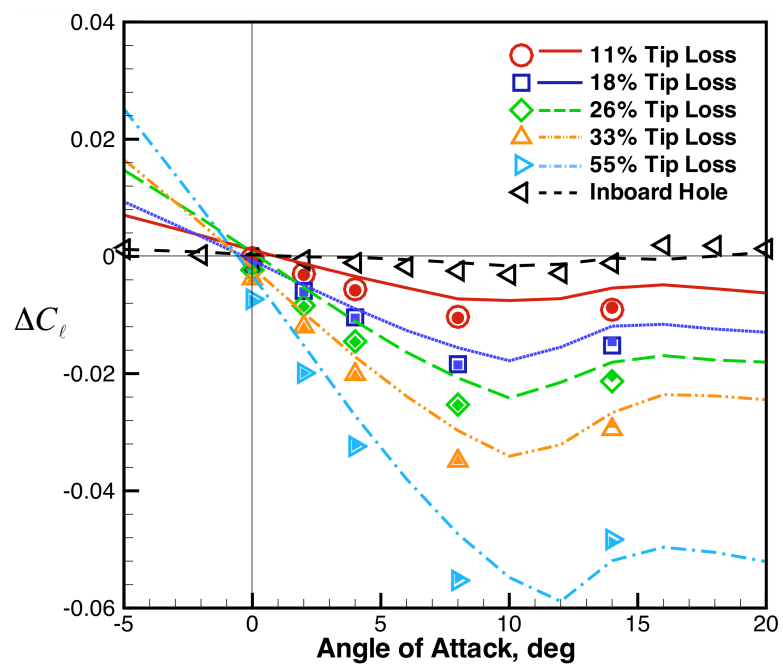

Figure 12. Incremental rolling moment due to left wing damage on GTM. $M=0.2, \beta=0$ deg. USM3D (open symbols), FUN3D (solid symbols), 14X22 WT experiment (line symbols).

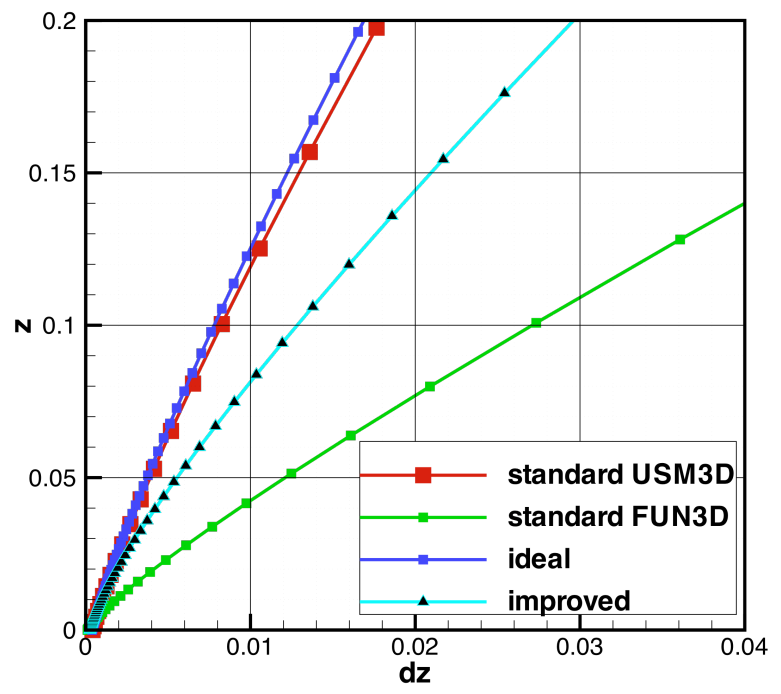

Figure A1. Wall normal mesh distribution within the viscous layers.

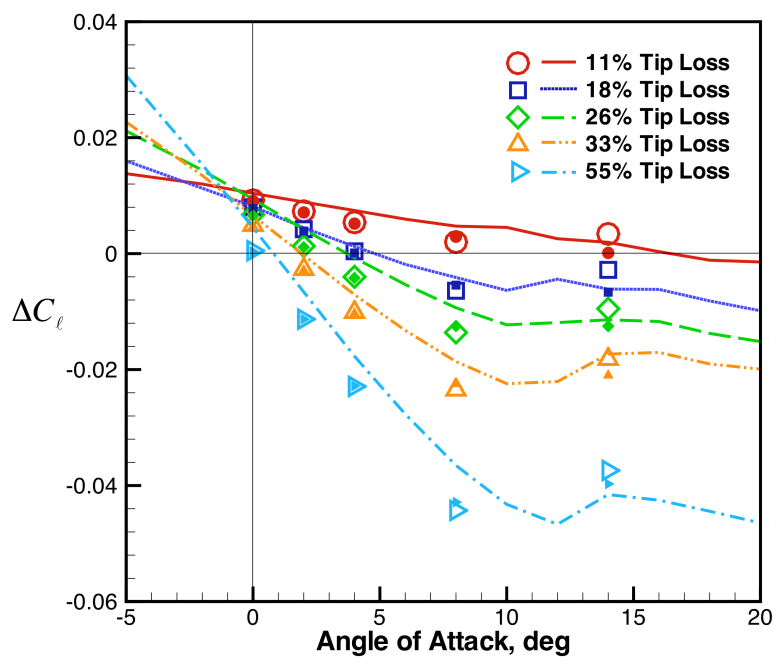

Figure 13. Incremental rolling moment due to left wing damage on GTM. M=0.2, $\beta=-4$ deg. USM3D (open symbols), FUN3D (solid symbols), 14X22 WT experiment (line symbols).

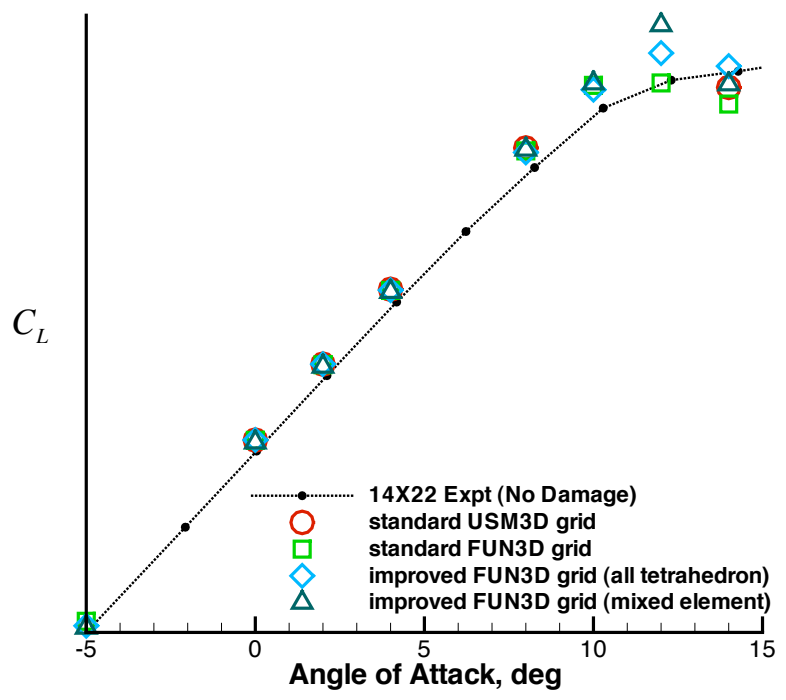

Figure A2. Effects of wall normal mesh distribution on solution accuracy. 